\title{
Improvements in the optical turbulence parameterization for 3D simulations in a region around a telescope
}

\author{
E. Masciadri ${ }^{1}$ and P. Jabouille ${ }^{2}$ \\ 1 Instituto de Astronomía, Universidad Nacional Autónoma de México, Apartado Postal 70-264, \\ 04510 México D.F. México \\ 2 Centre National des Recherches Météorologiques, Météo France, 42 avenue G. Coriolis 31057 Toulouse, France
}

Received 14 February 2001 / Accepted 1 June 2001

\begin{abstract}
In preceding papers we have presented comparisons between simulated and measured $C_{N}^{2}$ profiles above two of the best astronomical sites: Cerro Paranal (Chile) (Masciadri et al. 1999a; Masciadri et al. 1999b) and Roque de los Muchachos (Canary Islands) (Masciadri et al. 2001a). These works aimed at validating the atmospheric model Meso-Nh which was conceived to simulate classical atmospheric parameters and was then adapted to simulate optical turbulence (3D maps of $C_{N}^{2}$ ). Good results were obtained but further improvements can be made. One of the principal limitations is the statistical reliability of the model that is, at the present time, not very high. In this paper we present some modifications introduced in the optical turbulence parameterization of the model that aim to reduce some systematic errors. Preliminary results, obtained comparing the simulations with measurements taken at the San Pedro Mártir site (Mexico) in March-April 1997, show that we are now able to obtain a better qualitative and quantitative estimate of the $C_{N}^{2}$ profiles. Finally, we show how we have been able to improve the spatiotemporal variability of the model.
\end{abstract}

Key words. atmospheric effects - methods: numerical

\section{Introduction}

The simulation of optical turbulence in a region around a telescope is based on a compromise. We need a sufficiently high horizontal and vertical resolution in order to describe the fine vertical distribution of the optical turbulence $\left(C_{N}^{2}\right.$ vertical profiles) and, at the same time, we need to simulate the optical turbulence over a surface with a linear size of, at least, a few tens of kilometers. Indeed, we have shown in the past that the turbulence produced by the friction of the atmospheric flow over the ground does not necessarily develop in situ and that it can propagate in horizontal directions affecting the atmosphere up to distances of the order of $10-20 \mathrm{~km}$. Moreover, in order to represent the whole troposphere in the vertical direction, we have to cover about $20 \mathrm{~km}$. So, in order not to exceed the available memory size for the computer runs, we have to find a numerical compromise that limits the maximum number of grid points in our atmospheric model and that describes, in the best way, the phenomenon that we want to describe: the optical turbulence. Previous studies (Masciadri et al. 1999a) showed that a good solution is to take a horizontal resolution of about $500 \mathrm{~m}$ extended

Send offprint requests to: E. Masciadri, e-mail: elena@astroscu.unam.mx over a surface of $60 \times 60 \mathrm{~km}$. In the vertical axis we have the first grid point at a height of $50 \mathrm{~m}$, a variable increasing grid size in the first kilometer above the ground and then a constant size of about $600 \mathrm{~m}$ until the end of the troposphere $(20 \mathrm{~km})$.

The optical turbulence parameterization used in the Meso-Nh model for such a configuration is described in a previous study (Masciadri et al. 1999a). The $C_{\theta}^{2}$ depends on the potential temperature gradient $\partial \theta / \partial z$ and on the dynamic turbulent scale $L$ :

$C_{\theta}^{2}=0.59 L^{4 / 3}\left(\frac{\partial \theta}{\partial z}\right)^{2} \phi_{3}$

where $\phi_{3}$ is an adimensional function that express the thermo-dynamic stability of the atmosphere. We refer the reader to (Masciadri et al. 2001a) for further information related to $\phi_{3}$. Finally, for $\lambda=0.5 \mu \mathrm{m}$, the $C_{N}^{2}$ is related to the $C_{\theta}^{2}$ by Gladstone's relation:

$C_{N}^{2}=\left(\frac{80 \times 10^{-6} P}{\theta^{2}}\right) C_{\theta}^{2}$.

We note that we cannot represent all the frequencies of the atmospheric turbulence spectrum. The model needs a minimum kinetic energy $\left(E_{\min }\right)$ to start a simulation. A system that starts with an energy $E=0$ does not evolve. 
What is the physical meaning of $E_{\min }$ ? We could associate this minimum energy $E_{\min }$ with a sort of background climatological noise. We have tried to measure it in order to prove our suggestion. Analyzing the $C_{N}^{2}$ profiles provided by balloons (which give a high vertical resolution for optical turbulence), we expected to find a background noise over which thin $C_{N}^{2}$ shears developed. In the balloon flights provided by different site testing campaigns we measured $C_{N}^{2}$ values as small as $10^{-20} \mathrm{~m}^{-2 / 3}$, which is the order of magnitude of the electronic noise of the detector. This means that it is not possible to discriminate $E_{\min }$ from the electronic noise, so that we cannot measure the $E_{\min }$ and our above suggestion cannot be verified.

The thermodynamical behavior of the atmosphere is defined by the Richardson number $R_{i}$ :

$R_{i}=\frac{g}{\theta} \frac{\partial \theta / \partial z}{(\partial U / \partial z)^{2}}$

where $g$ is due to gravity acceleration, $\theta$ the potential temperature and $U$ the horizontal wind velocity. If $R_{i} \leq 0.25$ the atmosphere flow is said unstable. If $R_{i}>0.25$ it is said stable. In other words, the stability depends on the gradient of $\theta$ and $U$. In the convective (or unstable) regime the value of $E_{\min }$ is rapidly overtaken during the simulation and the outputs of the model are independent of the value chosen for $E_{\min }$. In the stable regime the evolution of the atmospheric flow is strongly dependent on the value of $E_{\min }$. Daytime is characterized principally by convective regimes, night-time principally by stable regimes. So, in our case, $E_{\min }$ is a free parameter that we have to fix. Figure 1 shows, as an example, the simulations of $C_{N}^{2}$ profiles obtained with the same model configuration but with two different values for $E_{\min }$.

In the regions in which the atmosphere is stable, different $E_{\min }$ values produce a stiff shift (see Fig. 1) of the $C_{N}^{2}$ profile obtained at the end of the simulation (3 hours). Indeed, in this case, the dynamic turbulent scale $L$ is approximately equal to the Deardoff scale:

$L=\sqrt{\frac{2 E}{\frac{g}{\theta} \frac{\partial \theta}{\partial z}}}$

Substituting Eq. (4) in Eq. (1) we find:

$C_{\theta}^{2}=0.20 \cdot \theta^{2 / 3}\left(\frac{\partial \theta}{\partial z}\right)^{4 / 3} E^{2 / 3} \phi_{3}$

and finally, using Eq. (2) and using the fact that for stable conditions (Masciadri et al. 2001a) $\phi_{3}$ is equal to 0.78 we obtain:

$C_{N}^{2}=3.35 \times 10^{-6} P^{2\left(1-2 R / c_{p}\right)} \theta^{-10 / 3}\left(\frac{\partial \theta}{\partial z}\right)^{4 / 3} E^{2 / 3}$

where $R / c_{P}=0.286$ and the pressure $P$ is given in $H P a$ units. $C_{N}^{2}$ then depends on the kinetic energy $E$ as:

$C_{N}^{2} \div E^{2 / 3} \rightarrow C_{N}^{2} \div E_{\min }^{2 / 3}$

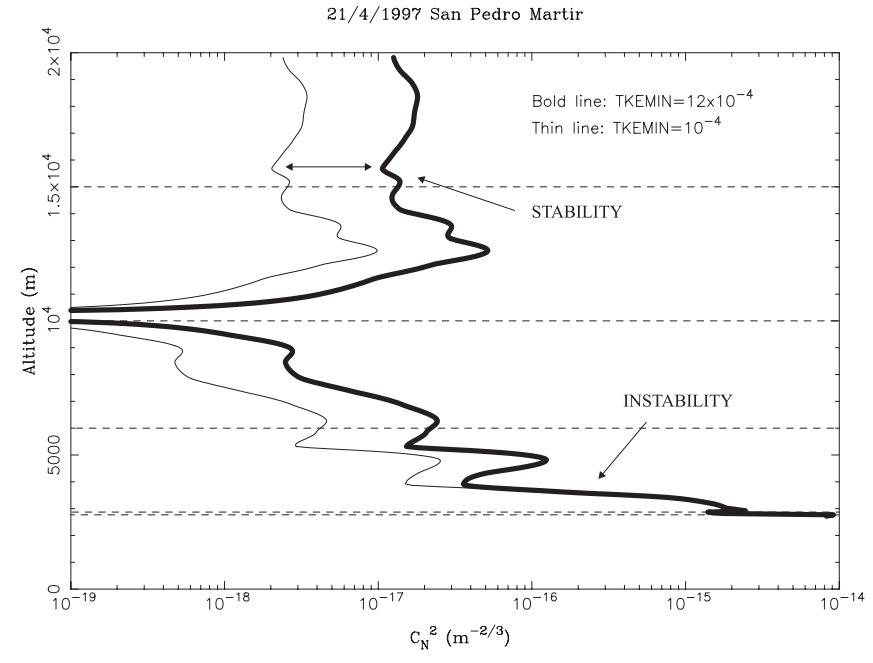

Fig. 1. Simulated $C_{N}^{2}$ vertical profiles above the San Pedro Mártir site the night of $21 / 4 / 1997$. Two different $E_{\text {min }}$ values are used. The bold line corresponds to $E_{\min }=12 \times 10^{-4} \mathrm{~m}^{2} \mathrm{~s}^{-2}$ and the thin line corresponds to $E_{\min }=10^{-4} \mathrm{~m}^{2} \mathrm{~s}^{-2}$.

The second equality results from the fact that $E \div E_{\min }$ for stable vertical profile of the atmosphere. In the regions characterized by a strong dynamic instability, on the contrary, no simple law relating $C_{N}^{2}$ and the kinetic energy $E$ exists. In the case shown in Fig. 1, an instability region is found in the low atmosphere, near the ground, where the turbulent activity is strong.

Because of the impossibility of measuring $E_{\min }$ we have to calibrate the model a posteriori comparing the measured and simulated optical turbulence. The purpose of the present paper is to describe this calibration of the model in detail.

We underline that, in the classic meteorological applications, the turbulence is treated in different ways for the boundary layer and for the clear air. Our problem is interesting from a numerical point of view because we want to describe, at the same time and with the same numerical solution, the turbulence developed in different parts of the atmosphere.

\section{Previous calibration techniques}

In the past we have tested several calibration techniques searching for an optimal procedure:

a. We tried to calibrate the model by comparing the measured and simulated seeing $(\varepsilon)$ over 8 nights in different intervals of simulation time (Masciadri et al. 1999b). We then computed the mean value of measured seeing $\left\langle\varepsilon_{\mathrm{SCI}}\right\rangle$ and the simulated seeing $\left\langle\varepsilon_{\mathrm{MNH}}\right\rangle$ over all of the nights. Finally the simulated seeing $\varepsilon_{i}$ was multiplied by the calibration coefficient $\alpha=\left\langle\varepsilon_{\mathrm{SCI}}\right\rangle /\left\langle\varepsilon_{\mathrm{MNH}}\right\rangle$. This solution is sensitive to the integral of the optical turbulence over the whole atmosphere, so that it does not give any information about the vertical distribution of the $C_{N}^{2}$. The same 

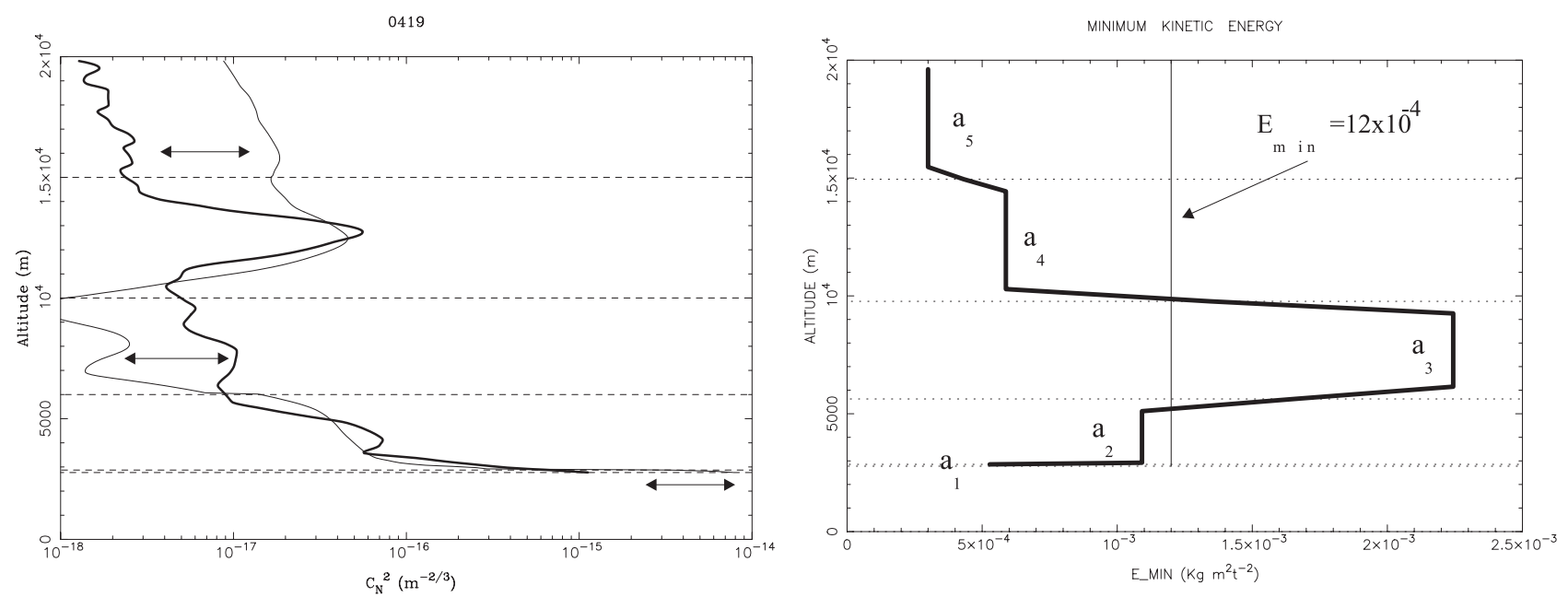

Fig. 2. On the left: measured $C_{N}^{2}$ profile provided by the Generalized Scidar (bold line) during the night of 19/4/1997 and the simulated $C_{N}^{2}$ profile provided by the Meso-Nh model the same night after 3 simulation hours (thin line). On the right: suggested vertical $E_{\min }$ profile to be used as initialization data. The parameters $\left(a_{i}\right)$ are to be estimated by a calibration procedure (see Sect. 3).

technique can also be applied to estimate the optimal simulation time, which we have found to be at least 3-4 hours. b. Another calibration technique was explored in the same paper (Masciadri et al. 1999b) in which measured and simulated $C_{N}^{2}$ profiles are compared for the same 8 nights. We suppose that we have, for each night $(m=1, M)$, a measured $C_{N}^{2}$ profile sampled over $N$ model levels $(i=$ $1, N): y_{m, i}$. For each night $m$ we have a simulated $C_{N}^{2}$ profile sampled over the same $N$ model levels: $x_{m, i}$. For each $m, y_{m, i}$ is the result of an average over the whole night and $x_{m, i}$ is the model output after a few simulation hours. We look for the $a_{m}$ value that minimizes the variance $\sigma_{m}^{2}$ defined as:

$\sigma_{m}{ }^{2}=\sum_{i=1}^{N}\left[y_{m, i}-a_{m} x_{m, i}\right]^{2}$,

and we then compute:

$a=\frac{\sum_{m=1}^{M} a_{m}}{M}$.

The simulated $C_{N}^{2}$ profiles are then modified as follows:

$C_{N}^{2 *}=C_{N}^{2} \cdot a$.

Using this calibration technique we obtained the following results: a linear correlation coefficient $r_{\mathrm{c}}=0.33$ between simulations and Scidar measurements and a $\sigma=0.54$ standard deviation between the Scidar measurements and the regression straight line between measurements and simulations. Moreover, the classical method of forecast-bypersistence (based on the assumption that the seeing $\varepsilon_{j}$ of the day $J$ is equal to the seeing $\varepsilon_{j+1}$ of the day $\left.(J+1)\right)$ gave $r_{\mathrm{c}}=0.20$ and $\sigma=0.35$. So, from a statistical point of view, the Meso-Nh model did not provide a result better than that provided by the forecast-by-persistence method. We proved that the principal reason for the low statistical reliability of the numerical technique was related to the low quality of the initialization data. Frequently, the radiosoundings that we used were not representative of the atmospheric flow over the site because the balloons were launched in a downstream position with respect to the principal wind direction, and in same cases, the distance between the meteorological station and the astronomic site was too large.

We also observed that, frequently, the results were similar to the ones shown in Fig. 2 (left side) obtained comparing simulations with $C_{N}^{2}$ measurements provided by the Generalized Scidar of the San Pedro Mártir campaign in 1997 (Avila et al. 1998). The bold line is the $C_{N}^{2}$ measured by the Generalized Scidar in 19/4/1997, the thin line is the $C_{N}^{2}$ profile simulated by Meso-Nh for the same night. $E_{\text {min }}$ has a value of $12 \times 10^{-4} \mathrm{~m}^{2} \mathrm{~s}^{-2}$. We observe that, in some regions, for example at $[10-13] \mathrm{km}$ and $[3.5-6] \mathrm{km}$, the profiles are well correlated. In other regions, for example at [6-10] km, above $15 \mathrm{~km}$ and near the ground, a stiff offset between the two profiles appears. This can be considered as a systematic error repeated in all the nights. So, it seems that it would be better to choose different values for $E_{\min }$ in different regions of the atmosphere. We should take a larger $E_{\text {min }}$ near the $[6-10] \mathrm{km}$ region and a smaller $E_{\min }$ above $15 \mathrm{~km}$ and near the ground. The optimized $E_{\text {min }}$ would have a variable vertical profile similar to the one shown in Fig. 2 (right side).

\section{A new calibration technique}

We now propose a new calibration procedure with the aim of reducing the systematic error intrinsically related to imposing a constant value of $E_{\text {min }}$ which we have detected in our previous studies. The principal steps of the calibration procedure are as follows:

1. using a constant $E_{\min }=12 \times 10^{-4} \mathrm{~m}^{2} \mathrm{~s}^{-2}$ value and comparing measured and simulated $C_{N}^{2}$ profiles of the 3 nights April 19, 20 and 21, 1997 (San Pedro Mártir site 
Table 1. Comparison between the measured and simulated seeing $\varepsilon$ during 3 nights (19, 20 and 21) on April 1997. The second column shows the parameters: seeing in the boundary layer $\left(\varepsilon_{\mathrm{BL}}\right)$, seeing in the free atmosphere $\left(\varepsilon_{\mathrm{FA}}\right)$ and the seeing in the whole atmosphere $\left(\varepsilon_{\text {Tот }}\right)$. The third column shows the seeing measured by the Generalized Scidar, the fourth column the seeing simulated using the "a posteriori" calibration procedure and the fifth column the seeing simulated by the model after the modification of $E_{\min }$ in the Meso-Nh code.

\begin{tabular}{ccccc}
\hline \hline NIGHT & Parameters & $\varepsilon_{\mathrm{SCI}}(\operatorname{arcsec})$ & $\varepsilon_{\mathrm{MNH}_{\mathrm{POST}}}(\operatorname{arcsec})$ & $\varepsilon_{\mathrm{MNH}}{ }_{\mathrm{PRE}}(\operatorname{arcsec})$ \\
\hline $19 / 4 / 1997$ & $\varepsilon_{\mathrm{TOT}}$ & 0.80 & 0.72 & 0.85 \\
$"$ & $\varepsilon_{\mathrm{BL}}$ & 0.59 & 0.58 & 0.63 \\
$"$ & $\varepsilon_{\mathrm{FA}}$ & 0.46 & 0.35 & 0.48 \\
\hline \hline $20 / 4 / 1997$ & $\varepsilon_{\mathrm{TOT}}$ & 0.75 & 0.71 & 0.74 \\
$"$ & $\varepsilon_{\mathrm{BL}}$ & 0.61 & 0.62 & 0.59 \\
$"$ & $\varepsilon_{\mathrm{FA}}$ & 0.36 & 0.26 & 0.36 \\
\hline \hline $21 / 4 / 1997$ & $\varepsilon_{\mathrm{TOT}}$ & 0.73 & 0.66 & 0.74 \\
$"$ & $\varepsilon_{\mathrm{BL}}$ & 0.60 & 0.53 & 0.50 \\
$"$ & $\varepsilon_{\mathrm{FA}}$ & 0.34 & 0.33 & 0.48 \\
\hline \hline
\end{tabular}

test campaign) we select 5 different regions of the atmosphere: [2771-2871] m, [2871-6000] m, [6000-10 000] m, [10 000-15 000] $\mathrm{m}$ and [15000-20000] $\mathrm{m}$ in which the turbulence has different behaviours. During the three nights the Generalized Scidar worked at the focus of the $2.1 \mathrm{~m}$ telescope in San Pedro Mártir,

2. we resample the measured and simulated $C_{N}^{2}$ profiles with the same vertical resolution: $50 \mathrm{~m}$,

3. for each night $m(m=1, M)$ we compute the $C_{N}^{2}$ average profiles provided by the Generalized Scidar measurements: $y_{m, i}$,

4. for each night $m$ and for each atmospherical region $k$ $(k=1,5)$ we compute:

$\chi_{m, k}^{2}=\sum_{j=1}^{P} \sum_{i=1}^{N}\left[a_{m, k} \cdot x_{m, i, j}-y_{m, i}\right]^{2}$,

where $P$ is the number of simulated profiles for each night. We have a $C_{N}^{2}$ simulated profile every $2 \mathrm{~min} . a_{m, k}$ is the free coefficient that has to be fixed minimizing the function $\chi_{m, k}^{2}$. The solution of the minimization is done by computing the coefficients:

$a_{m, k}=\frac{\sum_{j=1}^{P} \sum_{i=1}^{N}\left(x_{m, i, j}\right) \cdot y_{m, i}}{\sum_{j=1}^{P} \sum_{i=1}^{N}\left(x_{m, i, j}\right)^{2}}$.

Finally we compute, for each $k$ :

$a_{k}=\frac{\sum_{m=1}^{M} a_{m, k}}{M} \quad k=1,5$

The $C_{N}^{2}$ vertical profile is then modified for each region:

$C_{N, k}^{2 *}=C_{N}^{2} \cdot a_{k} \quad k=1,5$.

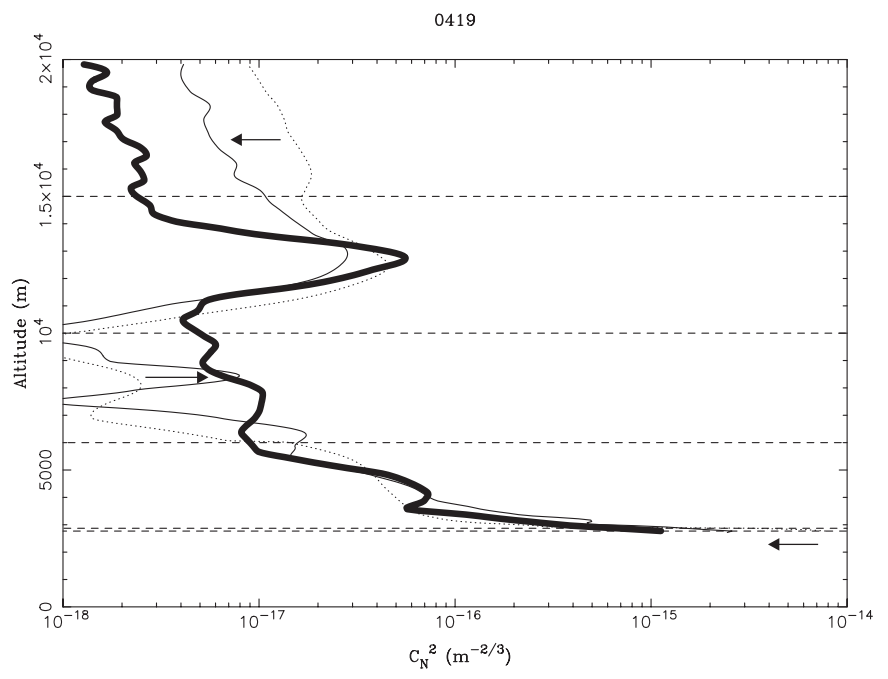

Fig. 3. Simulated and measured $C_{N}^{2}$ vertical profiles on the 19/4/1997 night. The bold line is measured by the Generalized Scidar. The dotted line is simulated with a constant $E_{\min }=$ $12 \times 10^{-4} \mathrm{~m}^{2} \mathrm{~s}^{-2}$ and the thin line is simulated with a variable $E_{\text {min }}$ vertical profile (Fig. 2, right hand side).

\section{Discussion}

Table 1 shows, for the three nights April 19, 20 and 21, 1997, the measured seeing (third column) and the simulated seeing (fourth column) obtained in the boundary layer $\left(\varepsilon_{\mathrm{BL}}\right)$, in the free atmosphere $\left(\varepsilon_{\mathrm{FA}}\right)$ and in the whole troposphere $\left(\varepsilon_{\mathrm{TOT}}\right)$. The simulated $C_{N}^{2}$ profiles are calibrated using the new technique presented in Sect. 3 and we have averaged the values over 3 simulation hours. We rejected (see Sect. 5) the first spurious part of the simulation resulting from the model adaptation to the ground. The boundary layer is defined as the first kilometer above the ground. We define a relative error as the following:

$\varepsilon_{\mathrm{rel}, \mathrm{POST}}=\frac{\left(\varepsilon_{\mathrm{TOT}, \mathrm{SCI}}-\varepsilon_{\mathrm{TOT}, \mathrm{MNH}-\mathrm{POST}}\right)}{\varepsilon_{\mathrm{TOT}, \mathrm{SCI}}}$ 


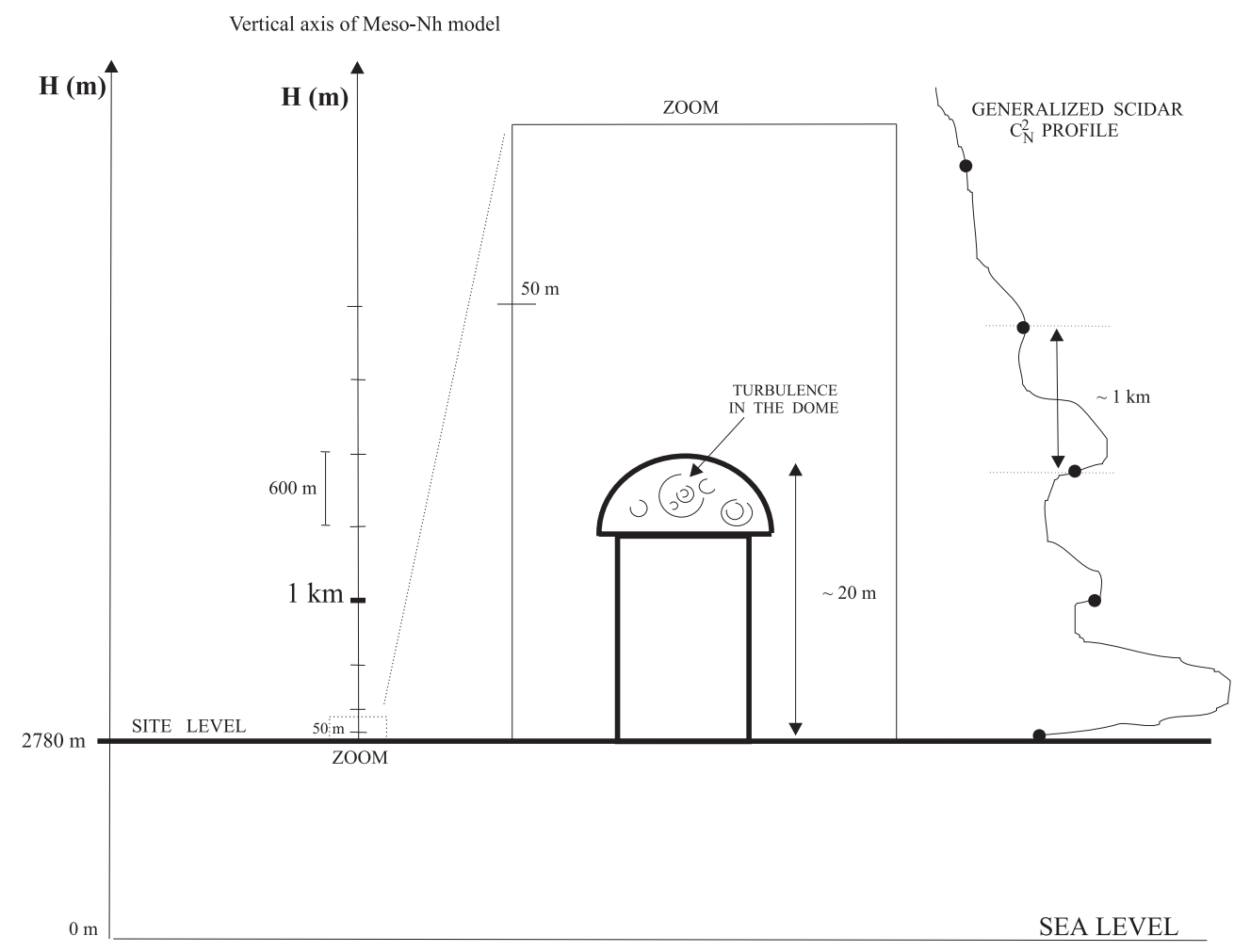

Fig. 4. On the left side is drawn the vertical axis of the altitudes above the sea level. Beside this one can see the vertical axis on which are sampled the grid points of the model Meso-Nh. The first grid point is at $50 \mathrm{~m}$, then a variable and increased size in the first $1 \mathrm{~km}$ and finally a constant size of about $600 \mathrm{~m}$ in the rest of the atmosphere. Beside this a zoom of the first few tens meters is shown. Correctly scaled the $2.1 \mathrm{~m}$ telescope in San Pedro Mártir is drawn inside the zoom. On the right side a typical vertical $C_{N}^{2}$ profile measured by a Generalized Scidar is drawn. The black dots mark the GS vertical resolution (about $1 \mathrm{~km})$.

where the values of the seeing are in the first line of each night in Table 1 . We calculate, for each night, $\varepsilon_{\text {rel,POST }}$ and then we calculate the average over all the nights. The relative error between the measured and the simulated $C_{N}^{2}$ profiles over the whole troposphere is estimated at about $8 \%$. Supported by this positive result we have tried to answer to the following question: if we modify $E_{\text {min }}$ over the whole $3 \mathrm{D}$ atmospheric model Meso-Nh using the $a_{k}$ found with the calibration procedure, can Meso-Nh reproduce the $C_{N}^{2}$ profiles with a statistical score equivalent to that obtained with the new "a posteriori" calibration that we have just presented?

Knowing the values of $a_{k}$ for $k=1,5$ (Eq. (13)), the kinetic energy is modified following:

$E_{\text {min }}^{*}=E_{\min } \cdot a_{k}^{3 / 2} \quad k=1,5$.

We can therefore retrieve the energy function shown in Fig. 2 (right side) and initialize the Meso-Nh model with this vertical cross section for $E_{\text {min }}$. In the last column Table 1 shows the values of the seeing simulated by the model after this modification in the different parts of the atmosphere: boundary layer, free atmosphere and the whole troposphere. The relative error calculated between the measurements and the new simulations (obtained replacing $\varepsilon_{\mathrm{TOT}, \mathrm{MNH}-\mathrm{POST}}$ with $\varepsilon_{\mathrm{TOT}, \mathrm{MNH}-\mathrm{PRE}}$ ) is estimated at about $3 \%$. This means that the statistical reliability of the model is not worse than in the case of a "a posteriori" calibration. Obviously, we intend to repeat this same test with a richer sample of nights but this preliminary result encourages us to follow this path. Moreover, we verified that the new calibration procedure reduces the systematic error that we discussed in the Sect. 2. As an example, Fig. 3 shows the $C_{N}^{2}$ profile simulated by the model in the night of 19/4/1997 after the modification of the $E_{\min }$ as Eq. (16). The bold line is the measured $C_{N}^{2}$ profile, the dotted line is the simulated $C_{N}^{2}$ profile obtained with $E_{\min }=12 \times 10^{-4}$ and the thin line is the simulated $C_{N}^{2}$ profile obtained initializing the model with the new $E_{\text {min }}$ which varies along the $z$-axis (Fig. 2 - right side). It is clear how the shape of the profile is changed in a suitable way: over the $15 \mathrm{~km}$ and near the ground the turbulence is reduced and in the range of $[6-10] \mathrm{km}$ is increased.

Let us now give some further commentaries related to the results shown in this paper. Figure 4 shows, on the left, the vertical sample of the actual model configuration. A zoom of the first $50 \mathrm{~m}$ is shown on the right part of the figure beside a suitably scaled telescope. The comparison between measurements and simulations in this region is particularly difficult because the model reproduces some turbulence that is not measured by the Scidar (the first meters corresponding to the height of the telescope which 

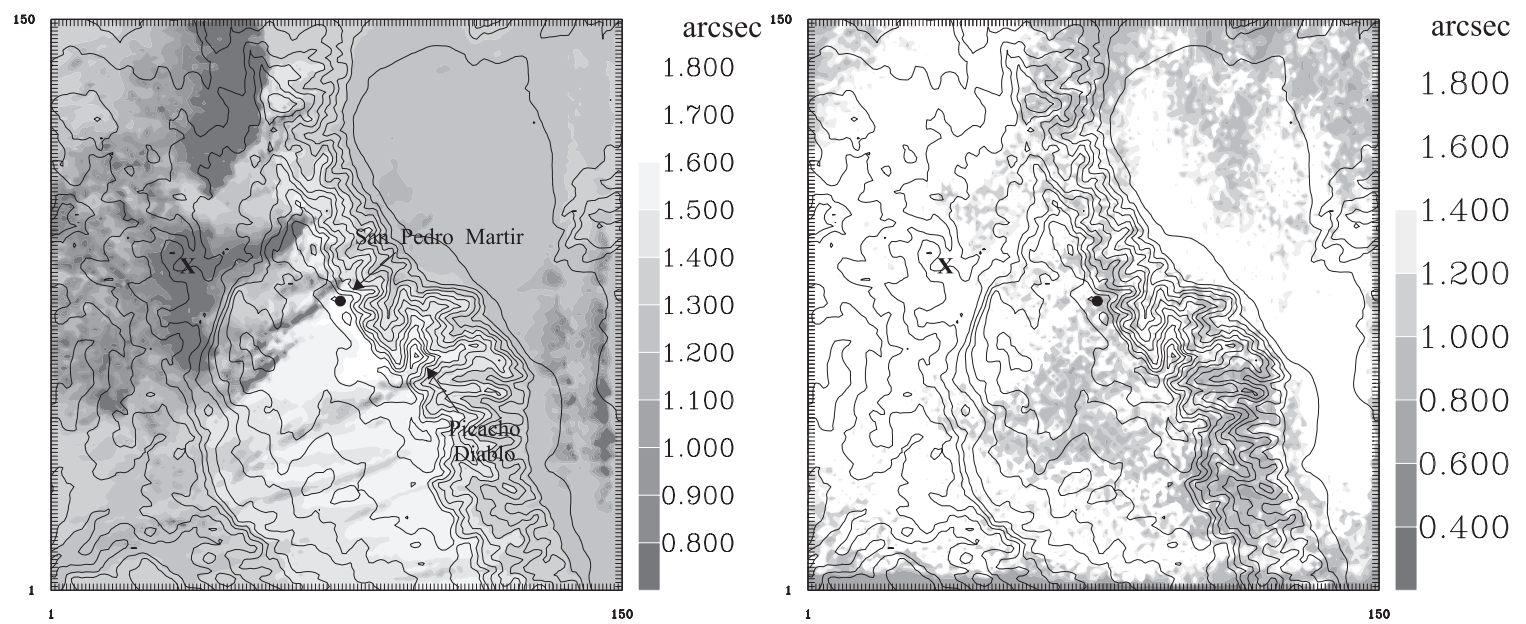

Fig. 5. Horizontal sections of the isoplanatic angle $\left(\theta_{\mathrm{AO}}-\right.$ on the left $)$ and the seeing $(\varepsilon-$ on the right) simulated above the San Pedro Mártir site over 3 hours (19/4/2000). The black isolines are the orographic lines. The color table shows large seeing and isoplanatic angle in white and small values in black. We note that a good site is characterized by a small seeing and a large isoplanatic angle.
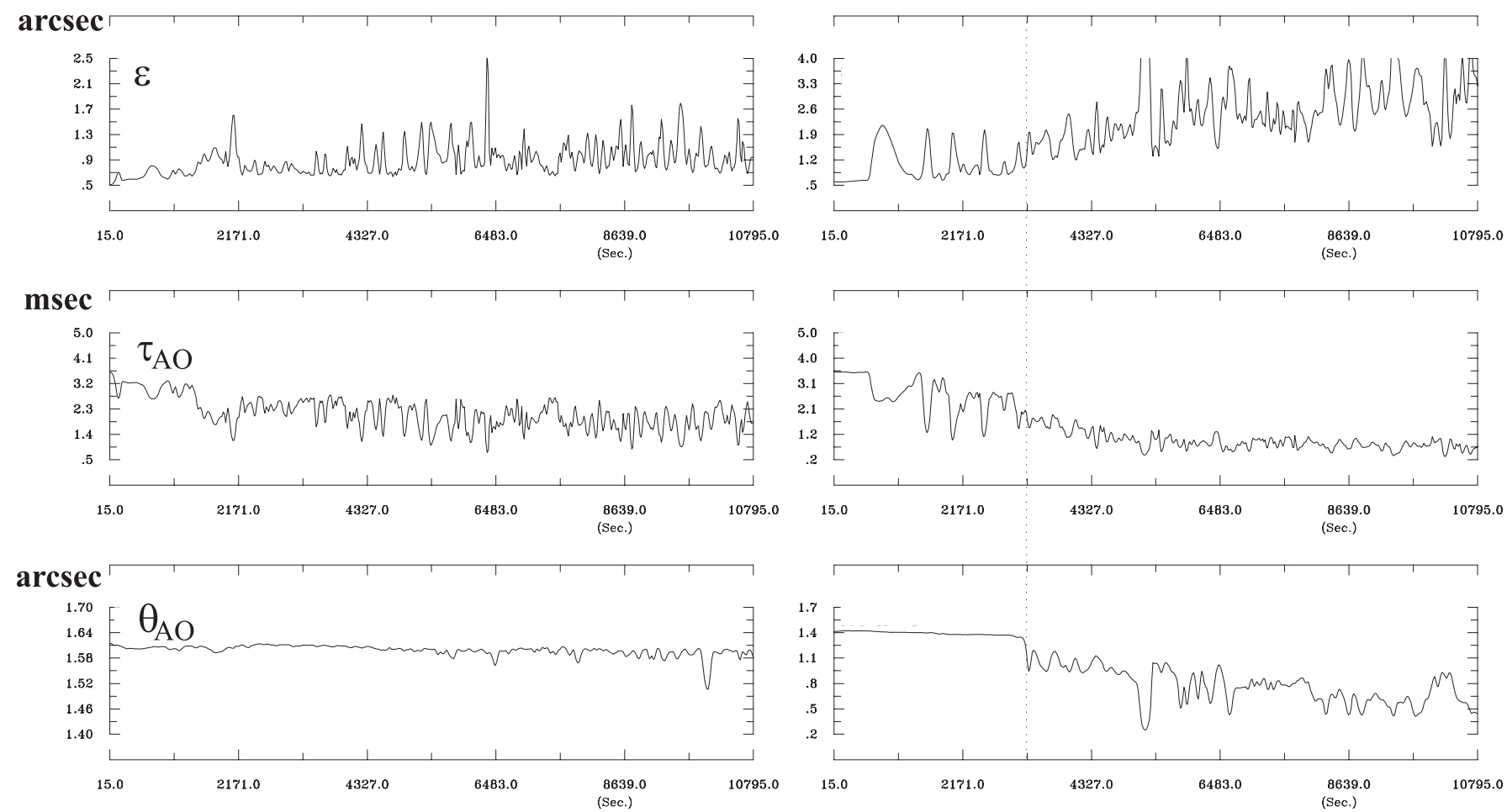

Fig. 6. Temporal evolution over 3 hours of some of the principal integrated astroclimatic parameters. From top to bottom: the seeing $(\varepsilon)$, the wavefront coherence time $\left(\tau_{\mathrm{AO}}\right)$ and the isoplanatic angle $\left(\theta_{\mathrm{AO}}\right)$. On the left the parameters are integrated above the place marked with a $d o t$, on the right above the place marked with a cross (see Fig. 5). We note that the vertical scales are not the same (in the seeing and isoplanatic angle case) for the cross and dot sites.

is about $20 \mathrm{~m}$ ) and the Scidar measures some turbulence that cannot be simulated by the model: the turbulence developed inside the dome. So, comparing the measured and simulated seeing in the boundary layer (third and fifth columns of Table 1) obtained in our study we can conclude that in the nights of April 19 the turbulence contribution provided by the first few meters above the ground is greater than that produced inside the dome, in the night of April 20 and 21 the contribution of the dome is greater than that developed in the first few meters. Anyway, we found differences in seeing estimations between measurements and simulations that are smaller than 0.1 arcsec in the boundary layer. This difference can be justified by the commentaries that we have just presented. It was estimated (Avila et al. 1998) that the dome seeing in the $2.1 \mathrm{~m}$ telescope was $\leq 1$ arcsec and measurements of the surface turbulence near the telescope (Sánchez et al. 2000) show a seeing smaller than 0.2 arcsec. 
We carried out some simulations using the first vertical grid mesh smaller than $50 \mathrm{~m}$, in order to approach the typical height of a telescope (about $20 \mathrm{~m}$ ) but the simulations broke down because of the stresses that develop near the strong ground slopes. This is due to the fact that, after such a change, the size of the first vertical grid box is strongly stretched and the optical turbulence parameterization used in Meso-Nh does not describe correctly the turbulence in such a region. The problem of modeling of the turbulence near the surface is not trivial and it was shown (Mason \& Thomson 1992; Andrén Brown et al. 1994; Sullivan et al. 1994) that the classic LES method consisting of directly resolving the greatest eddies and parameterizing the part of the turbulence not resolved with an eddy viscosity does not necessary give good results. New ideas and suggestions relating to the modelization of the turbulence near the ground are currently being actually studied for both LES and mesoscale models (Redelsperger et al. 2000). In this last paper, preliminary results obtained in neutral and convective regimes appear to agree with recent measurements of the turbulence spectrum near the ground. Results in strong stable conditions (such as the ones we are simulating) have not yet been obtained. We therefore consider that, in the current state of affairs, $50 \mathrm{~m}$ is an acceptable height for the first vertical grid mesh.

\section{Spatiotemporal variability}

In a previous paper (Masciadri 2001b), we have shown an example of the temporal evolution of the $C_{N}^{2}$ calculated at different model levels and we have proven that the model has a good variability at least in the first $10 \mathrm{~km}$. In particular we showed that the optical turbulence simulated by the model during time changes from its initial condition. This has to be considered as a fundamental asset of such a model in the context of site testing. What about the integrated parameters? Figure 5 shows two horizontal sections of the seeing ( $\varepsilon$, on the right), and the isoplanatic angle $\left(\theta_{\mathrm{AO}}\right.$, on the left), obtained after 3 simulation hours above the San Pedro Mártir site. The surface extends to a $60 \mathrm{~km} \times 60 \mathrm{~km}$ horizontal domain and the site is at the centre of the figure. The same $C_{N}^{2}$ shown in Masciadri (2001b) (Fig. 2) is used to calculate the integrated parameters. Figure 6 shows the temporal evolution of the integrated parameters $\left(\varepsilon, \tau_{\mathrm{AO}}\right.$ and $\left.\theta_{\mathrm{AO}}\right)$ calculated above the dot and the cross points shown in Fig. 5. $\tau_{\mathrm{AO}}$ is the wavefront coherence time. On the left are shown the parameters integrated above the dot and on the right the ones above the cross. We observe that:

- the first simulation hour is to be rejected because the model is adapting to the ground. This is evident on the right of Fig. 6. This means that the first simulation hour is not representative of the atmospheric state;

- the mean values (averaged over time) of the seeing $(\varepsilon)$, the isoplanatic angle $\left(\theta_{\mathrm{AO}}\right)$ and the wavefront coherence time $\left(\tau_{\mathrm{AO}}\right)$ are substantially different above the

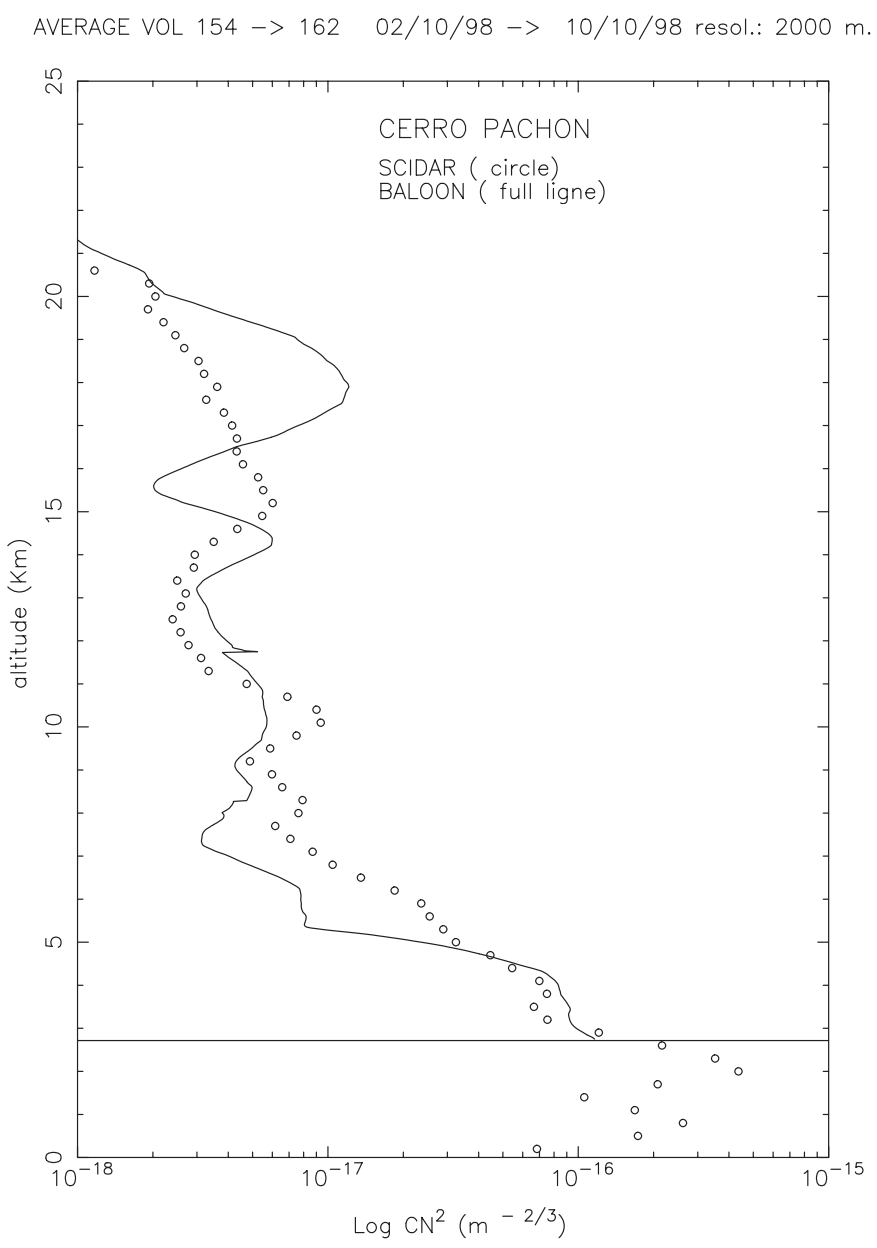

Fig. 7. Vertical $C_{N}^{2}$ profiles provided by the Generalized Scidar (dots) and balloons (line) during 9 nights (2-10 October) at Cerro Pachón on 1997 site testing campaign. The profiles are the results of an average over all the nights.

two places. In this way, the model Meso-Nh is shown to be a viable tool for discriminating between different sites;

- in Fig. 5, on the left, it is evident that the small region having the largest isoplanatic angle (white color) extends around the San Pedro Mártir site and the Picacho del Diablo, which are the two highest peaks in the region of the Sierra de Baja California. Figure 5, on the right, shows an irregular distribution of the seeing in the region around these two peaks. This is due to the fact that the turbulence has different intensities near the ground, to which the isoplanatic angle is less sensitive. In this case, the better isoplanatic angle is due to a higher altitude of the two peaks than the surroundings.

\section{Conclusions}

In this paper we propose a new calibration procedure and we present some modifications implemented in the Meso$\mathrm{Nh}$ code. We find that:

- the new calibration procedure reduces some of the systematic errors that had been detected in the previous 
version of the model. The relative error between the measurements and the simulations is now quantitatively not larger than $8 \%$ and the shape of the measured $C_{N}^{2}$ profiles is better reproduced by the simulated ones. This result is particularly interesting if we think that the improvements obtained at high altitudes ( $h \geq 15 \mathrm{~km})$, for example, will permit us to obtain better model estimations of the isoplanatic angles (which are particularly sensitive to the high altitude distribution of the optical turbulence).

- knowing the $a_{k}$ coefficients provided by the new calibration procedure, we can reconstruct a new $E_{\min }(z)$ function (replacing the previously used, constant value for $\left.E_{\min }\right)$. We have shown that, if the model is initialized with this new $E_{\min }$ function, the systematic errors are reduced.

- recent modifications of the Meso-Nh code permit us to have new outputs of the model such as the temporal evolution of the principal integrated astroclimatic parameters: the seeing $\varepsilon$, the wavefront coherence time $\tau_{\mathrm{AO}}$ and the isoplanatic angle $\theta_{\mathrm{AO}}$. We have shown that the variability of the model is good from a temporal and spatial point of view and that the model can discriminate sites characterized by a different optical turbulence.

We intend to repeat, in the near future, the same study with a larger number of nights. The challenge is to keep the statistical validity of the model at values as small as those obtained in this paper. A forthcoming study using the $C_{N}^{2}$ profiles provided by the Generalized Scidar on 16 nights (San Pedro Mártir campaign on May 2000) is planned. The current version of such an instrument permits a discrimination of the dome seeing from the boundary layer seeing $\varepsilon_{\mathrm{BL}}$. Moreover, during this site-testing campaign, measurements of the surface optical turbulence were done using an instrumented mast (Sánchez et al. 2000). We think that, with these data, we shall be able to give a more precise estimation of the reliability of the model near the surface.

We conclude by underlining that the calibration system presented in this paper is based on a comparison between simulations and measurements. The measurements are taken as a reference, that is as the most representative estimation of the real state of the atmosphere. It is desirable to have a good agreement between the measurements taken by different instruments at each altitude in order to have a good calibration. Any systematic differences obtained using different instruments have to be eliminated or at least reduced. Otherwise we will have to arbitrarily decide which kind of measurements are to be used for the model calibration. For example, in Fig. 7 we show the $C_{N}^{2}$ profiles provided by the Generalized Scidar and the balloons averaged over 9 nights $(2-10)$ on October 1997 on Cerro Pachon (Chile) (Vernin et al. 2000). It is evident that the two instruments measure a different turbulence above $15 \mathrm{~km}$ and that the model calibration can give different results if we use the Scidar or the balloon measurements.

It may be possible to define a more general method to calibrate the model. The optimal solution would be to find a theoretical justification for a universal $E_{\min }$ vertical profile depending on the atmospheric characteristics. In order to obtain such a result, we will need many studies done over many astronomical sites.

Acknowledgements. This work was supported by the TIM (Telescopio Infrarojo Mexicano) Project. We thank P. Bougeault for kindly supplying CPU time for this study. We are grateful to all the people of Bougeault's team for their complete availability in supporting us in the handling of the Meso-Nh model. We thank J. Vernin and R. Avila for kindly giving us the $C_{N}^{2}$ profiles measured in San Pedro Mártir. The work of E. Masciadri was supported by the CONACYT grant J32412E and the DGAPA grant IN118199.

\section{References}

Andrén Brown, A. R., Graf, J., Mason, P. J., et al. 1994, Quart. J. Roy. Meteorol. Soc., 120, 1457

Avila, R., Vernin, J., \& Cuevas, S. 1998, PASP, 110, 1106

Masciadri, E., Vernin, J., \& Bougeault, P. 1999a, A\&AS, 137, 185

Masciadri, E., Vernin, J., \& Bougeault, P. 1999b, A\&AS, 137, 203

Masciadri, E., Vernin, J., \& Bougeault, P. 2001a, A\&A, 365, 699

Masciadri, E. 2001b, Astronomical Site Evaluation in the Visible and Radio Range, ASP Conf. Ser., Marrakech, 13-17 November 2000

Mason, P. J., \& Thomson D. J. 1992, J. Fluid. Mech., 242, 51

Redelsperger, J. L., Mahé, F., \& Carlotti, P. 2000, Bound. Layer Meteor.

Sánchez, L., Avila, R., \& Masciadri, E. 2000, Astronomical Site Evaluation in the Visible and Radio Range, ASP Conf. Ser., Marrakech, 13-17 November 2000

Sullivan, P. P., McWilliams, J. C., \& Moeng, C. H. 1994, Bound. Layer Meteor., 71, 247

Vernin, J., et al. 2000, Gemini Report

Web Page: http://www.gemini.edu/sciops/instruments/ adaptiveOptics/ 